\title{
EL TRASTORNO DEL ESPECTRO AUTISTA EN LA UNIÓN EUROPEA (ASDEU)
}

\section{Autism Spectrum Disorders in the European Union (ASDEU)}

\author{
Manuel Posada de la Paz \\ Instituto de Salud Carlos III. Instituto de Investigación de Enfermedades Raras \\ mposada@isciii.es \\ ORCID: 0000-0002-8372-4180 \\ Ricardo CANAL-BEDiA \\ Universidad de Salamanca. Instituto Universitario de Integración en la Comunidad
}

Recepción: 27 de julio de 2020

Aceptación: 18 de octubre de 2020

Resumen: La mejora de los servicios de apoyo para las personas con trastorno del espectro autista (TEA) y a sus familias en Europa requiere conocer su realidad epidemiológica, así como identificar las experiencias y procedimientos para la detección, diagnóstico, tratamiento y apoyo más eficaces y mejor valorados por los receptores de estos servicios. El proyecto "El Trastorno del Espectro Autista en la Unión Europea" (ASDEU) ha impulsado entre 2014 y 2018 un conjunto de estrategias de investigación que han permitido aplicar un modelo de salud pública sobre los TEA centrado en los métodos de estimación de la prevalencia, los programas de detección precoz y la mejora del diagnóstico y la intervención temprana. En este artículo se presenta un resumen de los resultados obtenidos, así como de las conclusiones principales. Se destaca la necesidad de contar con un sistema fiable de medición de la prevalencia de los TEA en la Unión Europea, siendo la versión metodológica más eficiente la basada en registros de TEA de base poblacional; así mismo se propone fortalecer las estrategias de detección para hacerla más sistemática a nivel poblacional; mejorar los procesos de diagnóstico para que sean más rápidos, incluyan información sobre las necesidades de apoyo de la persona e incorporen a las familias; y potenciar la intervención temprana con mayor participación de las familias y vinculación al entorno habitual del menor.

Palabras clave: autismo; TEA; prevalencia; detección, diagnóstico, intervención temprana. 
АвSTRACT: The improving support services for people with autism spectrum disorder (ASD) and their families in Europe requires knowledge of their epidemiological status, as well as the identification of the most effective experiences and procedures for detection, diagnosis, treatment and support that are most valued by the recipients of these services. The project “Autism Spectrum Disorder in the European Union” (ASDEU) has promoted between 2014 and 2018 a set of research strategies that have allowed the application of a public health model to ASD focused on prevalence estimation methods, early detection programs and the improvement of diagnosis and early intervention. This article presents a summary of the results obtained, as well as the main conclusions. It highlights the need to set up a comprehensive system based on ASD population registries, which provides a systematic information about the ASD prevalence in Europe; likewise, the article proposes to strengthen detection strategies to make it more systematic at a population level; to improve diagnostic processes so that they are faster, include information on the support needs of the person and incorporate families; and to promote early intervention with greater participation of families and linkage to the child's regular setting.

KEYwORDs: autism; ASD; prevalence; screening, diagnosis, early intervention.

\section{Introducción}

$\int 1$

L TRASTORNO DEL ESPECTRO AUTISTA (TEA) es una alteración del neurodesarrollo que aparece en la infancia, cuyas dos manifestaciones principales son los déficits en comunicación social y el comportamiento restrictivo y repetitivo, acompañados a menudo de anormalidades en el procesamiento sensorial. El trastorno perdura toda la vida, presentando una amplia variabilidad intra- e interindividual en la expresión de los síntomas tanto en cuanto al momento de inicio, como al modo en que evolucionan a lo largo del desarrollo (Asociación Americana de Psiquiatría APA, 2013). No se ha establecido una etiología concreta del TEA, aunque se asume que su origen está en la interacción de una amplia variedad de factores genéticos y ambientales (Modabbernia et al., 2017).

El TEA es una de las alteraciones neuroevolutivas de aparición temprana más frecuentes. Además, su prevalencia ha aumentado drásticamente en las últimas décadas, pasando de un caso por cada 5.000 niños en 1975 a uno cada 45 (2,24\%) en 2014 ${ }^{1}$, lo que supuso un aumento del $700 \%$ en un plazo de 40 años (Zablotsky et al., 2017). Ese incremento continuo ha sido motivo de preocupación para familias, organizaciones, servicios y responsables políticos en el contexto europeo y en todo el mundo. Por eso, las organizaciones han venido abogando ante muchos gobiernos nacionales de toda la Unión Europea (UE) por mejorar servicios que deben dar respuesta a esta demanda creciente. Sin embargo, aunque en algunos países de la UE se han realizado estudios de prevalencia, no hay mecanismos para obtener información de manera sistemática y fiable sobre la tendencia de la prevalencia del TEA a nivel europeo, lo cual supone

1 En 2014 fue el año en el que se presentó el proyecto ASDEU.

Ediciones Universidad de Salamanca / CC BY-NC-ND

Siglo Cero, vol. 52 (2), 2021, abril-junio, pp. 43-59 
un obstáculo importante para el desarrollo de políticas apropiadas y ajustadas a las necesidades en cada región de la Unión.

Otro problema importante vinculado al autismo es que las familias experimentan la aparición de síntomas de TEA en uno de sus miembros como un acontecimiento muy preocupante que generalmente perturba el bienestar personal y familiar (Hayes y Watson, 2013). En consecuencia, a partir de ese momento comienza un proceso de adaptación, generalmente complejo y largo, en el que tienen que afrontar apresuradamente retos como recibir un diagnóstico formal, acceder a un tratamiento adecuado y adaptar la dinámica familiar e individual a esta nueva realidad del autismo en la familia. Entre los aspectos que se han identificado como contribuyentes a esta pérdida de bienestar (además de los factores individuales asociados a las características de los niños y sus padres), hay factores contextuales relacionados con el entorno social y la disponibilidad de servicios de apoyo profesional. Por ejemplo, muchas familias con un niño pequeño con TEA informan de dificultades para acceder a los servicios, altos costes asociados y falta de información y apoyo durante el proceso de diagnóstico (Hodgetts et al., 2015; Kogan et al., 2008; Thomas et al., 2016; Wang et al., 2013); así que no es de extrañar que una de las principales fuentes de estrés parental reportadas por los padres de niños con TEA sea la falta de apoyo profesional adecuado (Bishop et al., 2007).

En relación con servicios prestados a personas con TEA en edad infantil, las fuentes de malestar e insatisfacción mencionadas por las familias son la demora por parte de los profesionales en abordar sus preocupaciones iniciales, el retraso en la obtención de un diagnóstico y la falta de apoyo profesional (Altiere y Kluge, 2009; Bishop et al., 2007; Bluth et al., 2013; Crane et al., 2016; Divan et al., 2012; Moh y Magiati, 2012; Osborne et al., 2008). También las dificultades de comunicación familia-profesionales pueden estar influyendo en el grado de insatisfacción expresado por algunas familias (Liptak et al., 2006), al igual que la ausencia de profesionales cualificados (Krauss et al., 2003) y la desorganización del plan de atención (Chiri y Warfield, 2012). Un estudio reciente llevado a cabo en Europa (Crane et al., 2018) sitúa esta problemática también en nuestro entorno. Ese estudio constató que los retrasos en el diagnóstico y la falta de comunicación entre la familia y los profesionales afectaban a la satisfacción con los servicios. Además, las familias esperaban más orientación, asesoramiento y apoyo emocional para ayudarles a comprender el significado y las implicaciones del diagnóstico que se dio a su hijo, y poder superar la crisis (Crane et al., 2018).

Con respecto a los adultos el panorama durante esta década que acabamos de terminar no ha sido mejor. Como en otras condiciones de inicio infantil, la mayoría de las personas con TEA se enfrentan a retos muy importantes cuando llegan a la edad adulta y una mayoría dependerá del apoyo de sus familias y comunidades durante toda su vida. Además, es posible que muchos hayan recibido el diagnóstico en la edad adulta y sus necesidades no sean bien comprendidas por los servicios de atención de adultos por no estar familiarizados con el autismo. Además, debido a la poca atención que las investigaciones han prestado a los adultos con autismo, realmente se sabe poco sobre los resultados a largo plazo a partir de los 18-20 años, así como sobre los factores que influyen en una vida satisfactoria y sobre cuáles son las mejores prácticas 
para el cuidado y el apoyo a los adultos con TEA y sus familias para lograr buenos resultados (Howlin et al., 2013; Magiati et al., 2014). La necesidad de mejorar nuestro conocimiento del autismo en la edad adulta es especialmente crítica, ya que el número de adultos autistas diagnosticados está aumentando a raíz del considerable incremento de casos desde la década de 1990 en el diagnóstico del autismo en niños.

\section{El camino iniciado en la UE}

Esta realidad tan compleja en el contexto europeo de desconocimiento de la dimensión real del problema desde un punto de vista epidemiológico de los servicios tanto para niños como para adultos requiere un esfuerzo de recogida de información en el marco de la UE que incluya también un análisis de las políticas sociales, sanitarias y educativas que apoyan a las personas con TEA existentes en los diferentes países. Un esfuerzo que promueva el desarrollo de un marco de trabajo europeo basado en la evidencia, de manera que se mejore la respuesta que se da al autismo en nuestro continente.

La UE no ha sido ajena a esta necesidad y, aunque el desarrollo de proyectos sobre el TEA en el marco de la Comisión Europea puede ser abordado desde diferentes ámbitos, ha sido fundamentalmente desde las Direcciones Generales de Empleo, la de Investigación y la de Salud, donde han surgido las tres principales iniciativas, aunque no las únicas (CORDIS, 2020).

Pero muchos de los proyectos desarrollados se han venido orientando a la búsqueda de explicaciones en el campo de la biología y la medicina o la psicología. El 2014 fue el año en el que se presentó el proyecto ASDEU. Hasta entonces muy pocos organismos habían intentado analizar la situación de las personas con TEA y sus familias en Europa con el objetivo de impulsar políticas homogéneas y equilibradas de mejora y promoción de servicios para toda la UE. Una de las primeras acciones fue financiada por la Dirección General de Salud de la Comisión Europea en el año 2006. Es el proyecto conocido por sus siglas en inglés EAIS (Sistema de Información Europeo en Autismo). Ese proyecto sentó las bases del desarrollo de un protocolo europeo para la medida de la prevalencia de los TEA en Europa, que sería más tarde recuperado y puesto en práctica, primero por el grupo de Irlanda (Boilson et al., 2016) y luego por otro proyecto europeo al que nos referiremos a continuación. Durante ese primer periodo se desarrollaron varias reuniones de paneles de expertos al amparo de la Dirección General de Salud, que culminaron en el proyecto cuyas siglas en inglés eran ENSAPC-Red Europea de Vigilancia del Autismo y la Parálisis Cerebral. Tras finalizar este proyecto en el año 2009, la financiación de proyectos y acciones sobre los TEA fue eliminada de los planes de acción de esa dirección general hasta el año 2013 en el que el propio Parlamento Europeo se interesó por el problema del autismo y sus familias y, tras dos intentos seguidos de proporcionar financiación para el estudio del TEA, finalmente consiguieron su objetivo. 
En el año 2014 el Parlamento Europeo aprobó una inversión en la investigación en autismo orientada a aspectos relacionados con la prevalencia y los costes del autismo en Europa, la detección e intervención precoz y la vida del adulto y del anciano.

Este proyecto fue gestionado por la Dirección General de Salud de la Comisión Europea, quien lanzó una licitación competitiva para recibir propuestas para el desarrollo de estos objetivos.

Un consorcio creado en España con la participación de 14 países europeos y 22 socios diferentes, procedentes de instituciones nacionales, y coordinados desde el Instituto de Salud Carlos III, fue el encargado de llevar a cabo el diseño de los métodos más apropiados y su desarrollo. El proyecto se tituló: Los Trastornos del Espectro del Autismo en la Unión Europea y se conoce por sus siglas ASDEU (título en inglés Autism Spectrum Disorders in the European Union), cuyos resultados resumidos se pueden encontrar en la página web http://asdeu.eu.

\section{Objetivos y estructura del estudio}

El proyecto se planteó dos objetivos:

1. Aplicar un modelo de salud pública al TEA centrado en los métodos de estimación de la prevalencia, los programas de detección precoz y la mejora del diagnóstico y la intervención temprana.

2. Crear un nuevo marco europeo basado en la evidencia que mejorará la atención tanto para los adultos como para las personas mayores con TEA.

Estos objetivos se centran en tres dimensiones principales para una vida saludable de las personas con TEA:

a) Estimación de la prevalencia y de la carga social y económica.

b) Detección precoz e intervención temprana del TEA.

c) Marco integral para el tratamiento y la atención de adultos con TEA incluyendo los aspectos relativos a los problemas de salud y otras dificultades particulares asociadas al envejecimiento.

Si bien en el proyecto original se desarrollaron todos estos objetivos, en este artículo nos limitaremos a describir los métodos de medición de la prevalencia del TEA y mostrar un resumen de los resultados, así como presentar la evaluación de los métodos y propuestas para la mejora de la detección precoz y la intervención temprana en Europa.

\section{Métodos}

El estudio de prevalencia utilizó como base metodológica el protocolo desarrollado por el proyecto EAIS antes mencionado, denominado European Prevalence Autism Protocol (EPAP) (Boilson et al., 2016). Básicamente este protocolo definía 
la edad a la que estimar la prevalencia, el lugar donde aplicar dicho procedimiento, la lista de instrumentos de medida para descartar o aceptar el diagnóstico de TEA y las fases del cribado de la población a estudiar. Dicho protocolo fue utilizado previamente en Irlanda, modificando algunas de sus bases para hacerlo más viable. En concreto en el ámbito del proyecto de ASDEU se utilizaron los siguientes métodos: 1) Ámbito geográfico: se eligieron 23 áreas geográficas distribuidas en 12 países europeos; 2) Edades del estudio: niños y niñas entre $7-9$ años; 3) Fases del estudio: dos, una a desarrollar en todas las escuelas y aulas del área geográfica definida y otra en el ámbito del centro de evaluación clínica/institución de cada socio del proyecto; 4) Herramientas de cribado: se adoptó el denominado "nominación del profesor", cuestionario utilizado en las escuelas para establecer una primera sospecha de posible diagnóstico de TEA; el SCQ, y el ADOS-G/ADOS2 para el diagnóstico; 5) Estrategias de implantación: los países se dividieron en tres estrategias diferenciadas (Tabla 1) basadas en su propia experiencia previa en el trabajo en epidemiología del autismo y en las condiciones éticas que sus propios comités les imponían; 6) Estimación de la prevalencia: las estimaciones de prevalencia se realizaron considerando el número de casos detectados y diagnosticados como TEA, siendo el denominador el número de casos que finalmente participaron en el estudio. Como factores de corrección, se incluyeron estimaciones sobre la ausencia de respuesta en cada estudio. La información se mostró en términos de prevalencia con los intervalos de confianza al $95 \%$.

\begin{tabular}{|l|l|l|}
\hline \multicolumn{3}{|c|}{$\begin{array}{c}\text { TABLA 1. Distribución de los métodos en función } \\
\text { de las áreas geográficas implicadas }\end{array}$} \\
\hline \multicolumn{1}{|c|}{ Método } & \multicolumn{1}{|c|}{ Países/áreas } & \multicolumn{1}{c|}{ Instrumentos } \\
\hline Base poblacional & $\begin{array}{l}\text { Dinamarca, Finlandia, } \\
\text { Islandia y dos regiones en } \\
\text { Francia }\end{array}$ & $\begin{array}{l}\text { Registros poblacionales } \\
\text { de casos de TEA }\end{array}$ \\
\hline $\begin{array}{l}\text { Cribado poblacional en } \\
\text { dos fases }\end{array}$ & $\begin{array}{l}\text { Polonia, Bulgaria, Pisa } \\
\text { (Italia), Portugal, País } \\
\text { Vasco (España), Rumanía }\end{array}$ & $\begin{array}{l}\text { Nominación del profesor; } \\
\text { SCQ; ADOS-G/ADOS2 }\end{array}$ \\
\hline $\begin{array}{l}\text { Cribado poblacional en } \\
\text { una fase }\end{array}$ & $\begin{array}{l}\text { Tres regiones de Italia, } \\
\text { Austria }\end{array}$ & SCQ; ADOS-G/ADOS2 \\
\hline
\end{tabular}

Todos los estudios de prevalencia fueron aprobados por los respectivos comités de ética nacionales o de las propias instituciones participantes.

En relación con los métodos para la mejora de la detección precoz y de la intervención temprana, el proyecto hizo un esfuerzo por conocer las prácticas eficaces que normalmente se utilizan en los diferentes países de la UE, incluyendo también el conocimiento desarrollado en nuestro contexto en relación a la identificación de parámetros no sociales y biomarcadores para el diagnóstico y la detección precoz. Las 


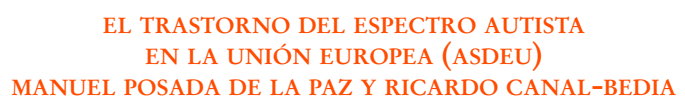

tareas llevadas a cabo utilizaron una combinación de estrategias metodológicas que se exponen a continuación.

\subsection{Revisiones de la literatura relacionadas con la detección precoz y con los métodos de intervención temprana para niños con TEA}

En las revisiones se tuvieron en cuenta experiencias europeas, tanto publicadas en revistas científicas o en documentos técnicos como las no publicadas. Específicamente para la identificación de experiencias de detección precoz, se seleccionaron los estudios publicados entre 1992 y 2015 que describieran con suficiente detalle una experiencia de detección precoz dirigida a la población en general y para el grupo de edad de 14 a 36 meses, aportando información sobre propiedades psicométricas de la herramienta y características del programa. Para la identificación de parámetros no sociales y biomarcadores para el diagnóstico y la detección precoz, la revisión sistemática seleccionó principalmente estudios prospectivos longitudinales con niños de alto riesgo desde el primer año de vida publicados entre 2005 y 2016.

En la revisión de experiencias de intervención temprana la búsqueda de estudios se dividió en dos grandes líneas de acuerdo con dos enfoques diferentes de intervención (Wong et al., 2015): modelos de tratamiento integral (CTM) que están diseñados para lograr un amplio impacto en el desarrollo del niño combinando diferentes técnicas; y modelos focalizados de intervención (FIP) que se usan para desarrollar una habilidad concreta (contacto visual, imitación, atención conjunta) o para lograr un aprendizaje específico. Es importante señalar que ambos enfoques de intervención incorporan o combinan métodos basados en el análisis conductual, la perspectiva evolutiva y técnicas relacionales. Ambos enfoques comparten también el propósito de desarrollar habilidades comunicativas, sociales y cognitivas (Narzisi et al., 2014; Odom et al., 2010). Otro criterio importante fue seleccionar solo experiencias que utilizaran procedimientos basados en la evidencia (Sackett et al., 2000).

Para la revisión sobre modelos CTM se realizaron dos búsquedas, una para ensayos controlados aleatorios y otra para ensayos controlados no aleatorios; ambas búsquedas cubrieron el marco temporal desde 2000 hasta 2017. Se obtuvieron 333 referencias (292 para ensayos controlados no aleatorios y 41 ensayos controlados aleatorios). Después del primer proceso de selección (cribado de datos duplicados) se seleccionaron 107 referencias para una revisión adicional.

Para la revisión de modelos FIP seleccionamos estudios que aportaran datos empíricos sobre resultados de tratamientos FIP, administrados a niños con diagnóstico de TEA de seis años o menos, que incluían intervenciones conductuales, de desarrollo o educativas para mejorar habilidades de comunicación e interacción social (imitación, contacto visual, atención conjunta, uso de gestos y juegos).

\subsection{Métodos de investigación cualitativa}

Los métodos de investigación cualitativa incluyeron grupos de discusión, encuestas en línea y entrevistas dirigidos a los principales implicados e interesados como 
son familias, profesionales de atención directa y dirigentes administrativos, tanto del ámbito de la salud, como el sanitario y el social.

En cuanto a los grupos de discusión, participaron 225 personas, incluyendo padres o madres de niños con TEA y profesionales de atención directa a niños o niñas menores de 6 años (pediatras, enfermeras, cuidadores y especialistas en intervención temprana). La distribución fue 146 (64\%) profesionales y 79 (36 \%) familiares. Los participantes pertenecían a diez países diferentes de la Unión Europea y se distribuyeron en veintinueve grupos de discusión, veinte grupos para el estudio de la detección y el diagnóstico tempranos y nueve grupos de discusión para la intervención temprana. El tamaño de los grupos osciló entre 5 y 11 participantes. Cada grupo de discusión fue dirigido por un facilitador y otro investigador que estuvo presente como asistente. Los temas tratados fueron la edad de acceso a los servicios; demoras en recibir los servicios y/o tratamientos y sus causas; la satisfacción con la atención y los tratamientos; los conocimientos que los participantes atribuían a los profesionales; limitaciones de los servicios; la participación de la familia; buenas prácticas; necesidades de capacitación que tienen los profesionales y las familias; la coordinación entre servicios, y procedimientos que conocen sobre detección, diagnóstico e intervención temprana.

Se analizaron las transcripciones obtenidas en los debates de los grupos de discusión, extrayendo y agrupándolos en categorías y ordenando las ideas, percepciones, preocupaciones e intereses expresados por los participantes. Este conjunto de categorías sirvió para elaborar las preguntas de la encuesta y organizar las encuestas diferenciando las preguntas dirigidas a las familias y las preguntas dirigidas a los profesionales. Siguiendo este procedimiento, se elaboraron dos encuestas diferentes para facilitar la organización de los datos de ambos grupos de encuestados (familias y profesionales) que tenían relación directa con un menor con TEA. El Comité de Bioética de la Universidad de Salamanca dio su aprobación y el cuestionario se tradujo a todos los idiomas de los países participantes. La encuesta fue distribuida a través de internet y respondieron a ella 2.032 participantes de 24 países diferentes.

Para el estudio sobre parámetros no sociales y biomarcadores también se hicieron entrevistas en las que se pidió a los encuestados que motivaran y aclararan sus respuestas. 237 profesionales que participaron en la encuesta general proporcionaron sus datos de contacto y fueron contactados por correo electrónico pidiéndoles que participaran en una entrevista de seguimiento. En total 16 clínicos de 8 países diferentes [Bélgica (3), Finlandia (1), Francia (2), Islandia (1), Italia (1), Polonia (2), Portugal (1) y España (5)] consintieron en realizar una entrevista por teléfono o por skype. Estos clínicos trabajaban en diversos entornos: hospitales (6), servicios de intervención temprana (3), centros especializados para el diagnóstico y la intervención de trastornos del desarrollo (3), servicios generales de salud mental (2), una escuela (1) y un gabinete privado (1) y tenían diferentes perfiles formativos: psicólogos (8), psiquiatras y neurólogos infantiles (4), profesores (2), un logopeda (1) y un terapeuta ocupacional (1). 


\section{Principales resultados y conclusiones}

El proyecto ASDEU ha supuesto un importante hito en el marco de la financiación de proyectos por parte de las organizaciones europeas. Si bien las direcciones generales más orientadas a investigación de carácter más general habían estado financiando estudios sobre los TEA y desde los programas marco europeos iniciales, solo un total de 131 han sido recogidos en la base de datos CORDIS (Comisión Europea, 2020) cuyo objetivo estuviese orientado al TEA. La mayoría de estos estudios iban dirigidos a las bases experimentales que pueden causar el que una persona nazca con un TEA, al margen de la edad del diagnóstico, pero ninguno de estos 131 proyectos aportaba la visión de la salud pública en el marco del autismo y sus repercusiones a lo largo de las edades de estas personas y de la propia carga social y familiar. A mediados de los años 2000, se abrió una luz de esperanza al financiarse por primera vez desde la Dirección General de Salud (DG SANTÉ) un proyecto que pudiese sentar las bases del problema del autismo y su relación con el estado de salud, la prevalencia y la carga social y familiar. Tras ese intento, la puerta de la DG SANTÉ se volvió a cerrar y tuvo que ser el Parlamento Europeo quien por dos años consecutivos insistiese en contribuir al conocimiento del autismo con la financiación de un proyecto cuyos objetivos y tareas venían marcados desde la propia Comisión Europea, dejando solo el espacio de la metodología a aplicar a los investigadores que obtuviesen dicha financiación. Así, la CE fijó la necesidad de desarrollar un estudio de prevalencia tomando como base la metodología del primer proyecto EAIS del año 2006 (Posada y Ramírez, 2008) en al menos 12 países europeos, la medición de los costes, el estudio de la situación de la detección e intervención temprana y la validez de los biomarcadores y el análisis de la vida del adulto y del anciano con TEA. Todo ello fue ensamblado en lo que finalmente ha constituido el proyecto ASDEU, cuyos resultados finales fueron presentados ante una comisión del Parlamento Europeo, ante las propias autoridades de la DG SANTÉ.

El estudio de prevalencia abarcó a 12 diferentes países europeos, 4 países completos: Dinamarca, Islandia, Finlandia y Rumania, y 19 regiones de países como Italia (4); Bulgaria (1); Polonia (4); Francia (2); Portugal (1); Austria (1); Irlanda (5); España (1). Los resultados basados en registros poblacionales (Delobel-Ayoub et al., 2020) muestran una prevalencia que oscila de 0,48 casos por cada 100 habitantes en uno de los registros de Francia, 0,73 en el otro registro francés, 0,77 en Finlandia, 1,26 en Dinamarca y 3,13 en el estudio de Islandia. Todos eran estudios basados en el total de la población, siendo los casos estudiados en Finlandia, Dinamarca e Islandia todos los niños de estos países en una franja de edades entre 7-9 años, mientras que los registros de Francia abarcaban también a toda la población, pero tenían un carácter regional.

De otra parte, los dos estudios publicados hasta la actualidad que utilizaron una metodología en dos fases (nominación del profesor en las aulas para un primer cribado y el SCQ para los que habían sido nominados por el profesor) presentaron una prevalencia del 1,15 \% en el estudio de Pisa (Narzisi et al., 2018) y de 0,59 en el estudio del País Vasco (Fuentes et al., 2020). Las cifras en el estudio de Bulgaria fueron muy bajas, 0,42 \% (Iskrov et al., 2018), y el resto de los estudios no publicados aún 
mostraban cifras que iban desde el 0,5 \% al 1,15\%. El conjunto de países y regiones que aplicaron solo un cribado de una fase utilizando el SCQ mostraron rangos de prevalencia del $1,27 \%$ al $1,79 \%$.

La variabilidad de las cifras de prevalencia observadas a lo largo de los estudios realizados en el total de países y regiones no es ajena a lo que ocurre en otros estudios donde se incluyen diferentes regiones o estados, como ocurre en el estudio del CDC donde las cifras entre los estados participantes oscilan entre 1,3 \% y 2,9 \%. En Europa el rango entre las cifras de los diferentes países ha sido mayor y para ello hay varias situaciones que lo pueden explicar. Mientras que en el estudio del CDC todos usaban la misma metodología, en ASDEU se implantaron inicialmente dos métodos con el objetivo de probar cuál era el que ofrecía más rendimiento para futuros estudios y seguimientos de la población. De esta manera se optó por incluir estudios de base poblacional, fundamentalmente basados en poblaciones no muy grandes, bien controladas desde hacía años, debido a la existencia de registros de autismo reconocidos por su amplia experiencia y datos que venían ofreciendo a lo largo de los años. Por el contrario, en aquellos países donde no existía esta infraestructura metodológica y donde tampoco existía una cultura amplia de medir la prevalencia, se optó por cribar en las escuelas en un rango de edad predeterminado y donde errores en el diagnóstico fuesen menores. De esta manera se optó por utilizar un instrumento usado previamente en EE. UU. (Hepburn et al., 2008) que consistía en que el profesor, basado en su amplio conocimiento de su alumnado, definiese qué alumnos y alumnas podrían tener rasgos compatibles con TEA. Este proceso era anónimo para los investigadores y solo tras revisar esas nominaciones se ofrecía a las familias la posibilidad de participar en la segunda fase, que consistía en rellenar el SCQ y, en caso de obtener una puntuación inferior al punto de corte reconocido para el SCQ, se evaluaba el caso mediante el ADOS-G o el ADOS2, según el centro evaluador.

Esta segunda metodología tuvo que desdoblarse en dos debido a que ciertos países no contaron con el consiguiente consentimiento informado para la fase de la denominación del profesor, lo que sin duda originó un tercer método que ampliaba el riesgo de encontrar diferencias en la prevalencia observada entre ellos. No obstante, el rango de cifras también se observó amplio entre los países que optaron por métodos similares. De los tres grupos, fue el de los estudios de base poblacional el que mostraba rangos más amplios, sobre todo por las cifras obtenidas en el estudio de vigilancia epidemiológica de Islandia.

El resto de la variabilidad observada se puede deber a múltiples factores que habitualmente coinciden en este tipo de estudios tan amplios. La ausencia de una participación de alumnos y profesores alta y sobre todo variable a lo largo de los colegios y aulas incluidas en el estudio puede, sin duda, llevar a sesgos de selección que condicionen el estudio. Este factor ha sido clave en el estudio del País Vasco y se ha controlado con estimaciones probabilísticas de los colegios y aulas que no participaron, sin que se haya visto muy alterado el resultado final obtenido con este ajuste y sin el ajuste probabilístico. Si bien esto lo podemos afirmar de este estudio concreto, no lo podemos asegurar en el resto de los estudios que usaron el cribado como herramienta de detección de casos de TEA en otros países. Otro problema que podría estar 
influyendo en las cifras de prevalencia es el método de diagnóstico. Si bien todos los estudios fueron llevados a cabo por profesionales con capacidad diagnóstica y manejo en el uso de las herramientas diagnósticas, no pudimos desarrollar estudios cruzados de casos para poder evaluar la reproducibilidad de los estudios entre profesionales de distintos países. La diferencia de idiomas a la hora de evaluar videos de niños en el proceso del ADOS era obvia y de ahí que, aunque se evaluó la posibilidad de realizar estudios prototipos con videos en inglés, no hubo posibilidades de llevar a cabo dicho tipo de estudios. En cualquier caso, el número de casos nuevos detectados en estos sistemas de cribado fueron pocos y un posible error en esta fase nunca hubiese podido producir esta variabilidad. En relación a los casos que ya estaban diagnosticados en niños y niñas dentro del rango de edad al tiempo del estudio, la reevaluación de los mismos fue desarrollada como una comprobación de la utilización de alguna de las versiones del ADOS en su proceso diagnóstico, pero nunca se tomó la decisión de reevaluar a todos esos niños.

Respecto a los estudios sobre detección precoz, diagnóstico e intervención temprana, los resultados de las revisiones sistemáticas están disponibles en Magán-Maganto et al. (2017), Bejarano et al. (2020) y Canu et al. (2020). Los resultados de la investigación cualitativa están disponibles en Bejarano et al. (2019) y en Iskrov et al. (2018).

El estudio sobre detección y diagnóstico tempranos indica que el cribado universal del TEA puede ayudar no solo a identificar a menores con este trastorno a una edad temprana, sino que además se identifican menores con otros problemas de desarrollo, por lo que también se estaría actuando sobre la detección de dificultades evolutivas de diferente tipo además del autismo, reduciendo la edad de diagnóstico en un considerable número de casos (Magán-Maganto et al., 2017).

Todas las experiencias sobre detección identificadas y revisadas en este proyecto han incluido estrategias de colaboración entre los sistemas públicos de salud, la atención temprana y las guarderías, lo que demuestra que la coordinación para la detección es posible y deseable, lo cual hace que la sensibilización y la capacitación de los profesionales en materia de TEA adquiera gran importancia. Sin embargo, en muchos de los estudios existe la limitación de que la recopilación de datos se ve afectada por bajas tasas de participación tanto de profesionales como de familias que aceptan participar, así como por el abandono de participantes antes de llegar al diagnóstico (MagánMaganto et al., 2017). Esto lleva a que los valores de prevalencia estimados tengan que ser interpretados con cautela, así como las propiedades psicométricas del programa.

Algunos de los programas de detección revisados incluyen varias etapas a diferentes edades y la mayoría están enmarcados dentro de los programas de vigilancia del desarrollo. En general la estrategia busca identificar el mayor número de casos de TEA y reducir el número de casos falsos negativos. Existen algunas herramientas de detección como el M-CHAT en sus dos versiones (Robins et al., 2001, 2014) que incluyen un procedimiento de verificación para minimizar el número de falsos positivos y así reducir el coste por mayor número de evaluaciones diagnósticas.

Hay muy pocos estudios sobre la eficacia del tratamiento de atención temprana con los niños detectados mediante programas universales de detección, por lo que aún no se puede afirmar con datos suficientes que los niños con TEA detectados 
precozmente mediante esos programas obtienen mejores resultados de tratamiento (Magán-Maganto et al., 2017). Además, sería un problema de orden ético importante detectar precozmente a niños con riesgo de TEA y proporcionar tratamiento solo a un subgrupo seleccionado aleatoriamente solo para demostrar que la detección precoz produce mejores resultados en los que reciben un tratamiento más precoz. Lo que deja claro este proyecto es que la detección temprana permite un acceso más rápido a mayores recursos y más especializados, como la intervención temprana, y eso tendría un impacto significativo en la mejora de las capacidades comunicativas y sociales del menor y, por tanto, se reducirá la sintomatología de TEA.

Los países en los que los profesionales que intervienen en las primeras etapas del desarrollo están mejor capacitados parecen ser los países en los que el trastorno se diagnostica más temprano, por lo que parece pertinente invertir en formación, pero esta formación debería ir acompañada de otras medidas como proporcionar herramientas, manuales de procedimiento y protocolos de actuación apoyados por regulaciones administrativas (Bejarano et al., 2020). Según los resultados obtenidos, estas estrategias han facilitado una percepción más favorable por parte de las personas implicadas (familias y profesionales) incrementando la coordinación entre instituciones. Si se lograra que las normas y regulaciones administrativas fueran dictadas a nivel europeo, se promovería la igualdad de acción entre los diferentes países.

A falta de un programa de cribado, los familiares (generalmente los padres) son quienes expresan las primeras preocupaciones de que pueda haber un problema en el desarrollo de su hijo, siendo los problemas de naturaleza comunicativa los signos que con más frecuencia empiezan a preocupar. Por su parte, aunque las herramientas de detección (generalmente cuestionarios) pueden reducir significativamente la edad de detección, a nivel europeo, solo un porcentaje muy pequeño de familiares indicó haber completado un cuestionario específico en algún momento del proceso de detección. Pero más de la mitad de los profesionales informaron que utilizan procedimientos sistemáticos de detección (Bejarano et al., 2020).

En cuanto a la demora en el acceso a los servicios, existe una correlación negativa entre el tiempo de detección y diagnóstico y la evaluación del proceso. Las familias de los niños que son detectados y diagnosticados más tarde tienden a evaluar el proceso de manera más negativa. Por tanto, es de vital importancia que los servicios atiendan a las familias lo antes posible, para que no se produzcan demoras y que una detección precoz vaya seguida de un diagnóstico tardío. Para ello, se sugiere el desarrollo de protocolos para reducir el tiempo entre detección y diagnóstico (Bejarano et al., 2020).

Los biomarcadores más prometedores para la detección de TEA están relacionados con aspectos estructurales del cerebro (mayor volumen total, amígdala más grande y diferencias estructurales en el cuerpo calloso). También se han estudiado, como posibles biomarcadores, la actividad cerebral atípica durante el habla, las diferencias en conectividad, deficiencias en la función inmunológica y algunos biomarcadores neonatales (hiperbilirrubinemia, ictericia y anoxia). Respecto a los signos tempranos no sociales más prometedores, se han propuesto los problemas de falta de atención, un peor desempeño en las tareas de procesamiento visual y el uso repetitivo de objetos a partir de los 12 meses; y un retraso en el desarrollo de la motricidad fina y gruesa a 
partir de los 14 meses. Los signos no sociales de TEA, especialmente en el dominio motor y del temperamento, ya se utilizan con bastante frecuencia en Europa. Pero el seguimiento ocular (por ejemplo, para evaluar la atención) es utilizado por menos de la mitad de los profesionales encuestados, a menudo no es una práctica común y solo la mitad de los encuestados lo considera factible. La mayoría de los encuestados consideran que es útil incluir signos no sociales en la detección temprana de los trastornos del espectro autista. Los biomarcadores solo son utilizados hasta ahora por una minoría de profesionales en Europa y no son considerados factibles por la mayoría. Sin embargo, alrededor de la mitad de los encuestados piensan que sería valioso utilizar los biomarcadores en la detección temprana de los TEA.

Respecto a la intervención temprana en Europa no se han realizado muchos estudios sobre la eficacia de la intervención temprana en niños pequeños con TEA en comparación con países como los Estados Unidos. Se han identificado 4 estudios en Europa y 32 que cumplan los criterios de la selección publicados en los Estados Unidos en los últimos 20 años (Magán-Maganto et al., 2017). Las prácticas de intervención que han demostrado mayor eficacia son las dirigidas al desarrollo de habilidades sociales y comunicativas con una metodología conductual administrada con técnicas naturalistas basadas en el conocimiento del desarrollo de la infancia temprana. Los estudios identificados han demostrado que el aumento de las horas de intervención o del número de sesiones puede producir beneficios en los efectos de la intervención, de modo que, cuantas más horas de tratamiento se proporcionen, mayor éxito se obtendrá, pero por el momento los datos no indican cuál es el número de horas necesarias para que esta mejora sea estadísticamente significativa (Bejarano et al., 2020).

La intensidad se incrementa con la participación de la familia. De hecho, la revisión sistemática indica que la participación efectiva de los padres en la intervención aumenta la eficacia del tratamiento (Cañete et al., 2018). Del mismo modo, si otros profesionales, como los maestros o los profesionales de la guardería, participan en la intervención, la eficacia mejora, pero en menor medida. Sin embargo, la mayoría de las familias informan de que tienen una participación esporádica o limitada en las actividades de tratamiento. La revisión sistemática nos ha permitido comprobar que, en los programas en que participan activamente los miembros de la familia, el tratamiento tiene mejores resultados. Además, la familia es un pilar básico porque generalmente acompaña a la persona con TEA durante casi toda la vida.

Por todo ello, es importante dar apoyo a los miembros de la familia desde el momento inicial no solo para lograr una mejor aceptación del problema, sino para que ayude a alcanzar los objetivos de la intervención lo antes posible. Se sugiere que este apoyo incluya, desde el momento de iniciar el tratamiento, un esfuerzo para definir un papel activo de la familia y para que se tenga en cuenta el contexto natural del niño. Los resultados indican también que las familias demandan que los servicios proporcionen información fiable sobre los diferentes métodos de intervención disponibles, así como recursos sociales y económicos para las familias que lo necesiten. 


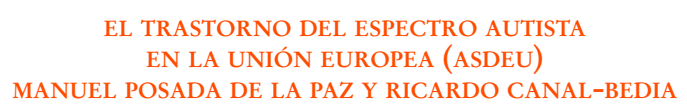

\section{Referencias bibliográficas}

Altiere, M. J. y Kluge, S. von. (2009). Searching for acceptance: challenges encountered while raising a child with autism. Journal of Intellectual and Developmental Disability, 34(2), 142-152. https://doi.org/10.1080/13668250902845202

APA. (2013). Diagnostic and Statistical Manual of Mental Disorders, 5th edn (1. ${ }^{a}$ ed., vol. 1-1). American Psychiatric Publishing.

Bejarano-Martín, Á., Canal-Bedia, R., Magán-Maganto, M., Fernández-Álvarez, C., Cilleros-Martín, M. V., Sánchez-Gómez, M. C., García-Primo, P., Rose-Sweeney, M., Boilson, A., Linertová, R., Roeyers, H., Van Der Paelt, S., Schendel, D., Warberg, C., Cramer, S., Narzisi, A., Muratori, F., Scattoni, M. L., Moilanen, I., ... y Posada de la Paz, M. (2019). Early detection, diagnosis and intervention services for young children with Autism Spectrum Disorder in the European Union (ASDEU): family and professional perspectives. Journal of Autism and Developmental Disorders. https://doi. org/10.1007/s10803-019-04253-0

Bejarano-Martín, Á., Canal-Bedia, R., Magán-Maganto, M., Fernández-Álvarez, C., Lóa-Jónsdóttir, S., Saemundsen, E., Vicente, A., Café, C., Rasga, C., García-Primo, P. y Posada, M. (2020). Efficacy of focused social and communication intervention practices for young children with autism spectrum disorder: a meta-analysis. Early Childhood Research Quarterly, 51, 430-445. https://doi.org/10.1016/j.ecresq.2020.01.004

Bishop, S. L., Richler, J., Cain, A. C. y Lord, C. (2007). Predictors of perceived negative impact in mothers of children with Autism Spectrum Disorder. American Journal on Mental Retardation, 112(6), 450-461. https://doi.org/10.1352/0895-8017(2007)112[450:POPN II]2.0.CO;2

Bluth, K., Roberson, P. N. E., Billen, R. M. y Sams, J. M. (2013). A stress model for couples parenting children with Autism Spectrum Disorders and the introduction of a Mindfulness intervention. Journal of Family Theory and Review, 5(3), 194-213. https://doi.org/10.1111/ jftr.12015

Boilson, A. M., Staines, A., Ramírez, A., Posada, M. y Sweeney, M. R. (2016). Operationalisation of the European protocol for autism prevalence (EPAP) for Autism Spectrum Disorder prevalence measurement in Ireland. Journal of Autism and Developmental Disorders, 46(9), 3054-3067. https://doi.org/10.1007/s10803-016-2837-y

Canu, D., Van Der Paelt, S., Canal-Bedia, R., Posada, M., Vanvuchelen, M. y Roeyers, H. (2020). Early non-social behavioural indicators of autism spectrum disorder (ASD) in siblings at elevated likelihood for ASD: a systematic review. European Child and Adolescent Psychiatry. https://doi.org/10.1007/s00787-020-01487-7

Cañete, M., Sánchez, M. ${ }^{a}$ C. y Corcho, P. (2018). Necesidades de apoyo percibidas por padres de niños con autismo entre 2-5 años, en México. Siglo Cero, 49(3), 75-93. https://doi. org/10.14201/scero20184937593

Chiri, G. y Warfield, M. E. (2012). Unmet need and problems accessing core health care services for children with Autism Spectrum Disorder. Maternal and Child Health Journal, 16(5), 1081-1091. https://doi.org/10.1007/s10995-011-0833-6

Comisión Europea. (2020). Servicio de Información Comunitario sobre Investigación y Desarrollo (CORDIS). https://cordis.europa.eu/es. Acceso el 01/06/2020.

Crane, L., Batty, R., Adeyinka, H., Goddard, L., Henry, L. A. y Hill, E. L. (2018). Autism diagnosis in the United Kingdom: perspectives of autistic adults, parents and

Ediciones Universidad de Salamanca / CC BY-NC-ND

Siglo Cero, vol. 52 (2), 2021, abril-junio, pp. 43-59

$$
-56-
$$




\section{EL TRASTORNO DEL ESPECTRO AUTISTA EN LA UNIÓN EUROPEA (ASDEU) \\ MANUEL POSADA DE LA PAZ Y RICARDO CANAL-BEDIA}

professionals. Journal of Autism and Developmental Disorders, 48(11), 3761-3772. https:// doi.org/10.1007/s10803-018-3639-1

Crane, L., Chester, J. W., Goddard, L., Henry, L. A. y Hill, E. (2016). Experiences of autism diagnosis: a survey of over 1000 parents in the United Kingdom. Autism, 20(2), 153 162. https://doi.org/10.1177/1362361315573636

Delobel-Ayoub, M., Saemundsen, E., Gissler, M., Ego, A., Moilanen, I., Ebeling, H., Rafnsson, V., Klapouszczak, D., Thorsteinsson, E., Arnaldsdóttir, K. M., Roge, B., Arnaud, C. y Schendel, D. (2020). Prevalence of Autism Spectrum Disorder in 7-9-yearold children in Denmark, Finland, France and Iceland: a population-based registries approach within the ASDEU Project. Journal of Autism and Developmental Disorders, 50(3), 949-959. https://doi:10.1007/s10803-019-04328-y

Divan, G., Vajaratkar, V., Desai, M. U., Strik-Lievers, L. y Patel, V. (2012). Challenges, coping strategies, and unmet needs of families with a child with Autism Spectrum Disorder in Goa, India. Autism Research, 5(3), 190-200. https://doi.org/10.1002/aur.1225

Fuentes, J., Basurko, A., Isasa, I., Galende, I., Muguerza, M. D., García-Primo, P., García, J., Fernández-Álvarez, C. J., Canal-Bedia, R. y Posada de la Paz, M. (2020). The ASDEU autism prevalence study in northern Spain. European Child and Adolescent Psychiatry. https://doi.org/10.1007/s00787-020-01539-y

Hayes, S. A. y Watson, S. L. (2013). The impact of parenting stress: a meta-analysis of studies comparing the experience of parenting stress in parents of children with and without $\mathrm{Au}$ tism Spectrum Disorder. Journal of Autism and Developmental Disorders, 43(3), 629-642. https://doi.org/10.1007/s10803-012-1604-y

Hepburn, S. L., Diguiseppi, C., Rosenberg, S., Kaparich, K., Robinson, C. y Miller, L. (2008). Use of a teacher nomination strategy to screen for autism spectrum disorders in general education classrooms: a pilot study. Journal of Autism and Developmental Disorders, 38(2), 373-382. https://doi.org/10.1007/s10803-007-0404-2

Hodgetts, S., Zwaigenbaum, L. y Nicholas, D. (2015). Profile and predictors of service needs for families of children with autism spectrum disorders. Autism, 19(6), 673-683. https:// doi.org/10.1177/1362361314543531

Howlin, P., Savage, S., Moss, P., Tempier, A. y Rutter, M. (2013). Cognitive and language skills in adults with autism: a 40-year follow-up. Journal of Child Psychology and Psychiatry, n/a-n/a. https://doi.org/10.1111/jcpp.12115

Iskrov, G., Decheva, G., Popova, E., Stefanov, R. y Posada de la Paz, M. (2018). Prevalence study on autism spectrum disorders in Bulgaria. Redki Bolesti i Lekarstva Siratsi, 9(2), 8-12. [In Bulgarian]. doi: 10.36865/2018.v9i2.6

Kogan, M. D., Strickland, B. B., Blumberg, S. J., Singh, G. K., Perrin, J. M. y Dyck, P. C. van. (2008). A national profile of the health care experiences and family impact of Autism Spectrum Disorder among children in the United States, 2005-2006. Pediatrics, 122(6), e1149-e1158. https://doi.org/10.1542/peds.2008-1057

Krauss, M. W., Gulley, S., Sciegaj, M. y Wells, N. (2003). Access to specialty medical care for children with mental retardation, autism, and other special health care needs. Mental Retardation, 41(5), 329-339. https://doi.org/10.1352/0047-6765(2003)41<329:ATSMCF $>2$ .0.CO;2

Liptak, G. S., Stuart, T. y Auinger, P. (2006). Health care utilization and expenditures for children with autism: data from U.S. national samples. Journal of Autism and Developmental Disorders, 36(7), 871-879. https://doi.org/10.1007/s10803-006-0119-9 


\section{EL TRASTORNO DEL ESPECTRO AUTISTA EN LA UNIÓN EUROPEA (ASDEU) \\ MANUEL POSADA DE LA PAZ Y RICARDO CANAL-BEDIA}

Magán-Maganto, M., Bejarano-Martín, Á., Fernández-Álvarez, C., Narzisi, A., García-Primo, P., Kawa, R., Posada, M. y Canal-Bedia, R. (2017). Early detection and intervention of ASD: a European overview. Brain Sciences, 7(12). https://doi.org/10.3390/ brainsci7120159

Magiati, I., TAy, X. W. y Howlin, P. (2014). Cognitive, language, social and behavioural outcomes in adults with autism spectrum disorders: a systematic review of longitudinal follow-up studies in adulthood. Clinical Psychology Review, 34(1), 73-86. https://doi. org/10.1016/j.cpr.2013.11.002

Modabbernia, A., Velthorst, E. y Reichenberg, A. (2017). Environmental risk factors for autism: an evidence-based review of systematic reviews and meta-analyses. Molecular Autism, 8, 13. https://doi.org/10.1186/s13229-017-0121-4

Moh, T. A. y Magiati, I. (2012). Factors associated with parental stress and satisfaction during the process of diagnosis of children with Autism Spectrum Disorders. Research in Autism Spectrum Disorders, 6(1), 293-303. https://doi.org/10.1016/j.rasd.2011.05.011

Narzisi, A., Costanza, C., Umberto, B. y Filippo, M. (2014). Non-pharmacological treatments in Autism Spectrum Disorders: an overview on early interventions for preschoolers. Current Clinical Pharmacology, 9(1), 17-26.

Narzisi, A., Posada, M., Barbieri, F., Chericoni, N., Ciuffolini, D., Pinzino, M., Romano, R., Scattoni, M. L., Tancredi, R., Calderoni, S. y Muratori, F. (2018). Prevalence of Autism Spectrum Disorder in a large Italian catchment area: a school-based population study within the ASDEU project. Epidemiology and Psychiatric Sciences, 6, 29, e5. https:// doi: $10.1017 /$ S2045796018000483

Odom, S. L., Boyd, B. A., Hall, L. J. y Hume, K. (2010). Evaluation of comprehensive treatment models for individuals with Autism Spectrum Disorders. Journal of Autism and Developmental Disorders, 40(4), 425-436. https://doi.org/10.1007/s10803-009-0825-1

Osborne, L. A., Mchugh, L., Saunders, J. y Reed, P. (2008). A possible contra-indication for early diagnosis of Autistic Spectrum Conditions: impact on parenting stress. Research in Autism Spectrum Disorders, 2(4), 707-715. https://doi.org/10.1016/j.rasd.2008.02.005

Posada, M. y Ramírez, R. (2008). Proposal for the European protocol for autism spectrum disorder prevalence (EPAP). http://ec.europa.eu/health/ph_information/dissemination/ diseases/docs/autism6.pdf

Robins, D. L., Casagrande, K., Barton, M., Chen, C. M. A., Dumont-Mathieu, T. y Fein, D. (2014). Validation of the modified checklist for autism in Toddlers, revised with followup (M-CHAT-R/F). Pediatrics, 133(1), 37-45. https://doi.org/10.1542/peds.2013-1813

Robins, D. L., Fein, D., Barton, M. L. y Green, J. A. (2001). The modified checklist for autism in Toddlers: an initial study investigating the early detection of autism and pervasive developmental disorders. Journal of Autism and Developmental Disorders, 31(2), 131-144.

Sackett, D. L., Straus, S. E., Richardson, W. S., Rosenberg, W. y Haynes, R. B. (2000). Evidence-based medicine: how to practice and teach EBM. Churchill Livingstone.

Thomas, R. E., Spragins, W., Mazloum, G., Cronkhite, M. y Maru, G. (2016). Rates of detection of developmental problems at the 18-month well-baby visit by family physicians' using four evidence-based screening tools compared to usual care: a randomized controlled trial. Child: Care, Health and Development, 42(3), 382-393. https://doi.org/10.1111/ cch. 12333

Wang, L., Mandell, D. S., Lawer, L., Cidav, Z. y Leslie, D. L. (2013). Healthcare service use and costs for Autism Spectrum Disorder: a comparison between medicaid and private 
insurance. Journal of Autism and Developmental Disorders, 43(5), 1057-1064. https://doi. org/10.1007/s10803-012-1649-y

Wong, C., Odom, S. L., Hume, K. A., Cox, A. W., Fettig, A., Kucharczyк, S., Brock, M. E., Plavnick, J. B., Fleury, V. P. y Schultz, T. R. (2015). Evidence-based practices for children, youth, and young adults with Autism Spectrum Disorder: a comprehensive review. Journal of Autism and Developmental Disorders, 45(7), 1951-1966. https://doi. org/10.1007/s10803-014-2351-z

Zablotsky, B., Colpe, L. J., Pringle, B. A., Kogan, M. D., Rice, C. y Blumberg, S. J. (2017). Age of parental concern, diagnosis, and service initiation among children with Autism Spectrum Disorder. American Journal on Intellectual and Developmental Disabilities, 122(1), 49-61. https://doi.org/10.1352/1944-7558-122.1.49 


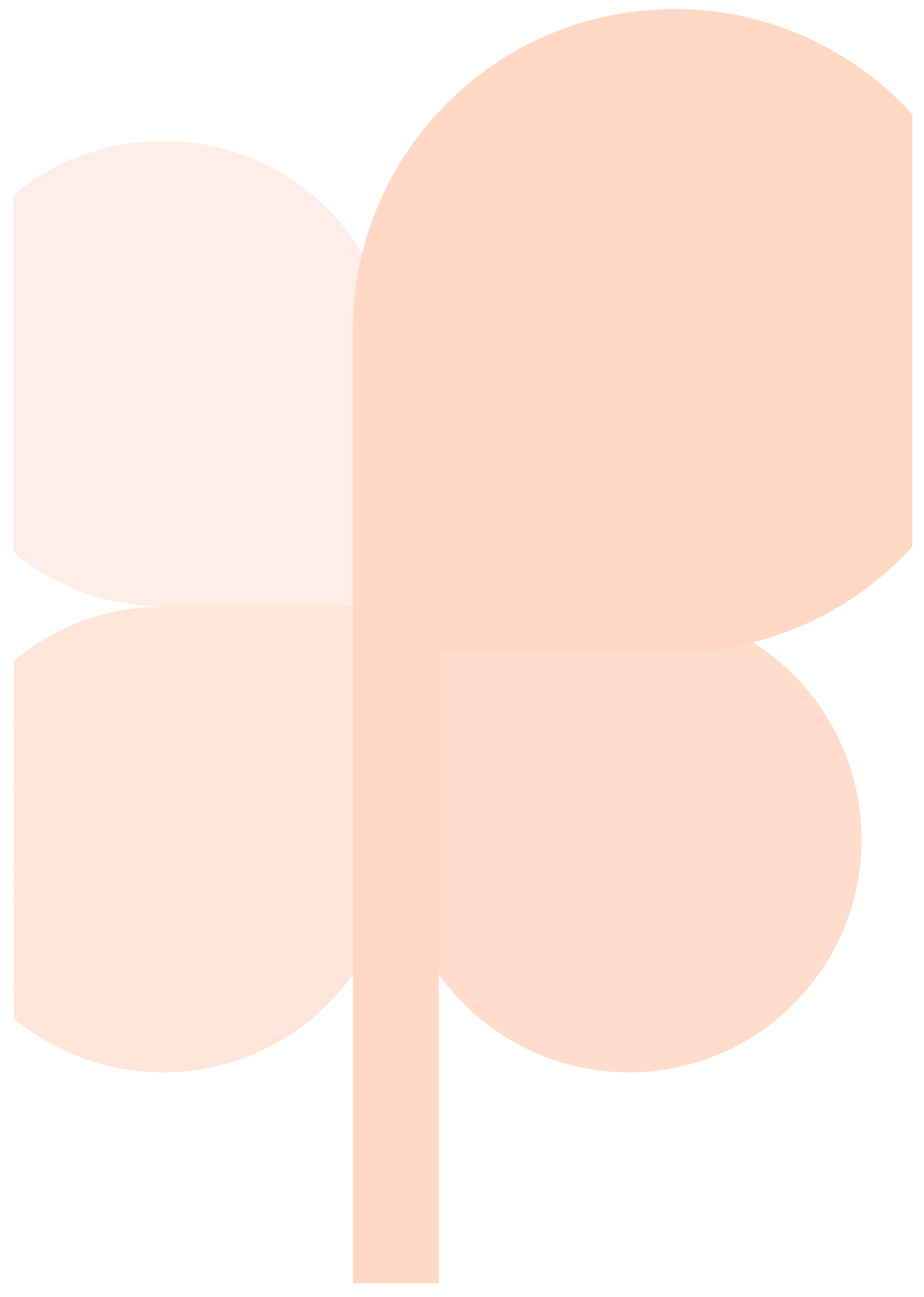

